\title{
ISOLATED VISUAL LOSS AND NEUROLOGICAL CAUSES: A HOSPITAL-BASED STUDY
}

\author{
G. Sree Ranga Lakshmi ${ }^{1}$, Rajesh Reddy², R. Ramesh ${ }^{3}$, Sita Ramu4, Radha Krishna ${ }^{5}$, A. Ashok Kumar 6
}

${ }^{1}$ Associate Professor, Department of Neurology, Osmania Hospital, Hyderabad.

${ }^{2}$ Consultant Neurologist, Department of Neurology, Apollo Hospital, Hyderabad.

${ }^{3}$ Assistant Professor, Department of Neurology, Osmania Hospital, Hyderabad.

${ }^{4}$ Assistant Professor, Department of Neurology, Osmania Hospital, Hyderabad.

${ }^{5}$ Consultant Neurologist, Department of Neurology, Care Hospital, Hyderabad.

${ }^{6}$ Consultant Neurologist, Department of Neurology, Tapadia Diagnostic Centre, Hyderabad.

\begin{abstract}
Visual loss is a common presenting symptom in the Outpatient Departments of Neurology, Ophthalmology and General Medicine disciplines. The present prospective study aims at evaluating 48 patients with partial or total visual loss as the predominant or only presenting symptom and with no associated ocular problems like cataract, glaucoma or diabetic retinopathy. The neurological causes were identified, analysed and tabulated; $39 \%$ of patients were below 20 years of age and there was 3:2 ratio of female versus male prevalence. Nearly $66 \%$ had acute visual loss and $35 \%$ had chronic visual loss. Majority of patients (48\%) had profound visual loss limited to finger counting; $6 \%$ had normal visual acuity associated with field defects. The visual evoked potentials were abnormal in all cases with acute and sub-acute presentation and in some patients with sudden or chronic presentation also. The VEP abnormalities consisted of prolonged P100 latencies, loss of amplitudes or poor wave morphology. Cranial CT scan was done in 36 patients and was abnormal in $83 \%$ patients. It showed intracranial tumours in $8 \%$ patients and infarcts in another $8 \%$ patients. Cranial MRI scan was done in 12 patients and was normal in $83 \%$ patients. The aetiological considerations and treatment protocols followed are discussed. The limitations of such study in the present setup are also highlighted.
\end{abstract}

\section{KEYWORDS}

Visual Loss, Visual Evoked Potentials, Demyelination, Optic Neuropathy.

HOW TO CITE THIS ARTICLE: Lakshmi GSR, Reddy R, Ramesh R, et al. Isolated visual loss and neurological causes: a hospital-based study. J. Evolution Med. Dent. Sci. 2016;5(69):5019-5024, DOI: 10.14260/jemds/2016/1140
INTRODUCTION
Visual impairment is a common symptom. Causes are generally ocular and include cataract, glaucoma, diabetes mellitus and nutritional deficiency (In children). ${ }^{1,2}$ Such cases are usually seen by ophthalmologists and are managed by them. Neurological causes of visual loss though are in a minority are important and may give good response if recognised early and managed properly. Many of these conditions are initially seen by ophthalmologists or general practitioners and as a result there may be delay in identifying and starting treatment. We are presenting our data showing the incidence of various categories of patients with visual impairment coming for neurological consultation and their outcome.
Majority of the cases have visual impairment due to anterior visual pathway disorders. History and clinical examination remain the crucial steps in identifying these diseases. The loss can be unilateral, bilateral or a field defect. Anterior visual pathways include retina, optic nerves and optic chiasm and the involvement results in moderate to profound visual loss along with impaired colour vision and loss of
The colour vision and pupillary reflexes are spared. More posterior lesions have congruous visual field defects. Relative Afferent Pupillary Defect (RAPD) is another good clinical sign to differentiate between anterior and posterior visual pathway defects, wherein the direct and indirect pupillary reflexes are elicitable and normal in the posterior visual pathway diseases. Most patients with acute visual loss can be given an aetiological diagnosis, while some of the chronic cases may not allow the cause to be identified and all the diagnostic tests may be normal. In some such cases, family history may be positive. At this point, it is worthwhile to remember the clinical difference between optic neuritis and papilloedema, and between papilloedema and pseudopapilloedema while going through the clinical details and arriving at a diagnosis. Another point of note is that patients having field defects have normal visual acuity in the field with intact vision, while the defective area is blind to the object tested resulting in an overall normal visual acuity. With the advent of sophisticated gadgets like MRI/CT brain scans and computerised Visual Evoked Potentials (VEP) testing, the diagnostic work-up has become easier, sharper and more focussed. pupillary reflexes. Posterior visual pathways include optic tracts, lateral geniculate body, optic radiation and occipital cortex. Their involvement results in field defects with relatively preserved visual acuity.

Financial or Other, Competing Interest: None.

Submission 28-06-2016, Peer Review 14-08-2016,

Acceptance 20-08-2016, Published 29-08-2016.

Corresponding Author:

Dr. G. Sree Ranga Lakshmi,

Associate Professor

Department of Neurology,

Osmania General Hospital,

Hyderabad, Telangana.

E-mail: rangalakshmi2000@gmail.com

DOI: $10.14260 /$ jemds/2016/1140

\section{MATERIAL AND METHODS}

\section{AIM OF THE STUDY}

To evaluate the neurological causes of visual loss in patients attending the tertiary care hospital in South India.

\section{Inclusion Criteria}

Patients presenting with unilateral or bilateral visual impairment of sudden, acute, sub-acute or chronic onset were included in the study.

\section{Exclusion Criteria}

1. Patients in whom the visual impairment is due to ocular cause were excluded. 
2. Patients in whom the visual impairment is associated with other neurological symptoms were also excluded.

\section{Presence of Headache was not an Exclusion Criterion}

This was a prospective study conducted from January 2004 to October 2006. Forty eight patients met the criteria and were included in the study. Majority of the patients were referred from Sarojini Devi Eye Hospital, Hyderabad. Some were directly from Neurology or from General Medical Outpatient Departments. All the patients were informed about the study and their consent was taken. Detailed history was taken regarding the mode of onset, progression of visual impairment and associated symptoms like pain. Clinical examination including fundus was carried out in all patients. Visual acuity was assessed by Rosenbaum's pocket screener and visual fields by confrontation method at the bedside.

The mode of onset of visual impairment was defined as sudden (Maximum severity within a few minutes), acute (Maximum severity within a few hours to 4 weeks), sub-acute (Progression from 4-8 weeks) and chronic (Progression beyond 8 weeks). Routine blood parameters were done in all the patients including HIV serology. Cerebrospinal Fluid (CSF) examination including biochemical analysis and cytology was done in 10 patients with chronic visual loss. CSF lactic acid levels were estimated in 4 patients. Cranial Computed Tomographic (CT) scan was done in 36 patients and Magnetic Resonance Imaging (MRI) was done in 12 patients. Patternshift visual evoked potentials were recorded in all patients. In the subjects whose visual acuity was so low that they could not concentrate on the monitor, the VEP test was done using goggles.

The patients who had acute demyelinating optic neuritis were treated with intravenous methylprednisolone 30 $\mathrm{mg} / \mathrm{kg} /$ day for 3 days followed by 11 days of oral prednisolone $1 \mathrm{mg} / \mathrm{kg} /$ day for 11 days as per the Optic Neuritis Treatment Protocol. ${ }^{3}$ All such patients were followed up for 6 months at regular intervals. The data was analysed and the results tabulated (Table).

\section{RESULTS}

Majority of the patients were in the category of 10-20 years' age group (19 patients; $39.58 \%$ ). There was only one patient above 50 yrs. of age indicating that visual impairment of neurological origin is predominantly a disease of younger age group.

Most studies showed a female-to-male ratio of 3:2, while the present study showed almost equal distribution.

Majority of the patients had acute visual loss (41.66\%), while $35.41 \%$ had a chronic variety of visual impairment. The remaining patients had sudden visual loss (8.33\%) and subacute visual loss (14.58\%). Sudden visual loss was seen in 4 patients due to stroke and consisted of visual field defects, either hemianopia or altitudinal defect. All patients belonging to this group were above 40 years of age and did not have any recovery during followup. Among the 4 patients with sudden visual loss, the fundi were normal in three and optic atrophy was present in one patient who had anterior ischaemic neuropathy in addition to an occipital infarct.

All the patients in the acute visual loss group (20 Patients) had optic neuropathy, 15 of them had unilateral and 5 patients had bilateral involvement.
In the category of sub-acute visual loss (7 patients) 5 patients had bilateral symptoms and 2 patients had unilateral symptoms, and all the cases were due to optic neuropathy. The optic neuropathy was in the form of papillitis in 16 patients with acute onset and 6 patients with sub-acute onset of visual loss. The remaining 4 patients with acute onset and one patient with sub-acute onset had normal fundus indicating retrobulbar optic neuritis. Chronic visual loss occurred in 17 patients of whom 15 (88.23\%) had bilateral visual loss, one had unilateral loss and the remaining patients had a bitemporal field defect.

Majority of the patients (47.95\%) had visual impairment limited to finger counting. Three patients (6.25\%) had normal visual acuity in the presence of field defects.

The VEPs were normal in $75 \%$ of patients with sudden visual loss and in $23 \%$ patients with chronic visual impairment. All the others had abnormal VEPs in the form of prolonged P100 latency, loss of amplitude or poor wave morphology or a combination of these features \{Fig $1 \& 2\}$.

Cranial CT scan was done in 36 patients and was normal in $83 \%$ subjects. The abnormalities were in the form of infarcts in $8 \%$ patients (Fig. 3) and tumours in another $8 \%$ patients pituitary adenoma, meningioma (Fig. 4) and subependymal mass (Fig. 5) in one case each. In some cases, the imaging abnormalities were not correlating with the visual loss. Cranial MRI scan was done in 12 patients and it was normal in the majority patients. The abnormal scan findings were noted in 2 patients only and consisted of pituitary tumour in one case and demyelination in the other case.

The aetiology of the visual impairment was stroke in the case of sudden visual loss (75\% patients), demyelinating optic neuropathy in the case of acute and sub-acute visual loss (100\% patients) and heterogeneous in the case of chronic visual loss. The causes varied from tumour in $23 \%$ patients, demyelination in $11 \%$ patients, familial in $11 \%$ patients and could not be identified in the remaining $54 \%$ patients with chronic visual impairment. In the other imaging, Cerebrospinal Fluid (CSF) analysis and serological tests were all negative. In 4 such patients muscle biopsy for ragged-red fibres was negative and in 3 others CSF lactate levels were normal suggesting the absence of mitochondrial inherited disorders.

\section{TREATMENT}

In cases with demyelinating optic neuropathy corticosteroids were administered, but dosage regimen differed between neurologists and ophthalmologists and also influenced partly by the patient's financial status. Three types of regimens were followed.2,3

1. Intravenous methylprednisolone 1 gm per day for 3 days followed by oral prednisolone for 11 days. It was given in 10 patients and all of them showed improvement by 6 months.

2. Intravenous dexamethasone - Twelve patients received intravenous dexamethasone for 3 days followed by oral prednisolone for 11 days. All the patients showed significant improvement at 6 months followup.

3. Oral prednisolone - Seven patients received oral prednisolone only for 14 days; 2 patients did not show any improvement at all even after 6 months. 


\begin{tabular}{|c|c|}
\hline Variable & No. of Patients \\
\hline Age: Below 40 yrs. & 40 \\
Above 40 yrs. & 8 \\
\hline Sex:Male & 23 \\
Female & 28 \\
\hline Mode of Onset: & 4 \\
Sudden & 20 \\
Acute & 7 \\
Sub-Acute & 17 \\
Chronic & 19 \\
Visual Loss: & 26 \\
Unilateral & 3 \\
Bilateral & 3 \\
Field Defect & 2 \\
Visual Acuity: & 23 \\
Normal (Field Defects) & 18 \\
Reduced & 2 \\
\hline Finger counting only & \\
Perception of light only & \\
No perception of light & Details of the Patients with \\
\hline Visual Impairment (Total No: 48)
\end{tabular}

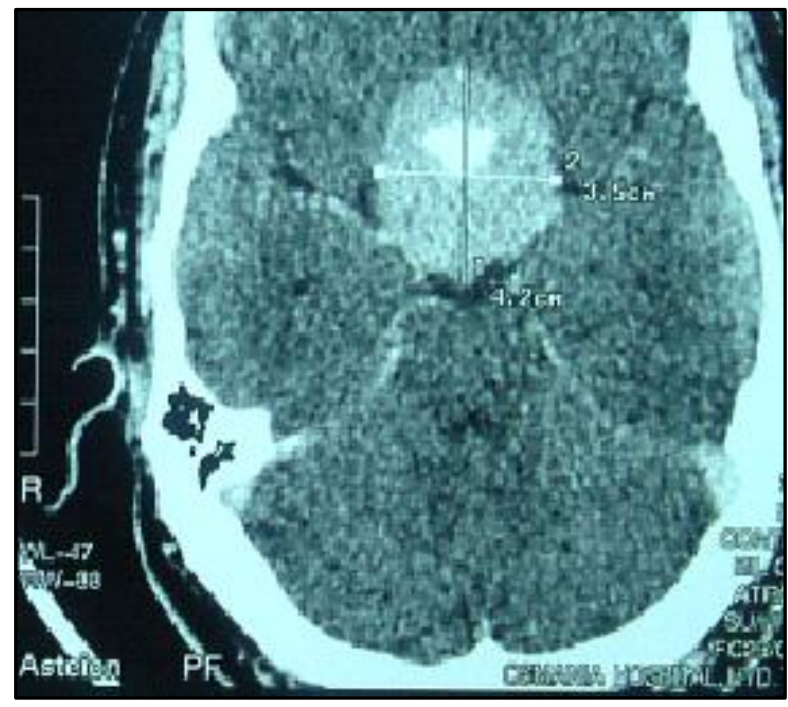

Fig. 4: CT Scan Brain showing Suprasellar Mass

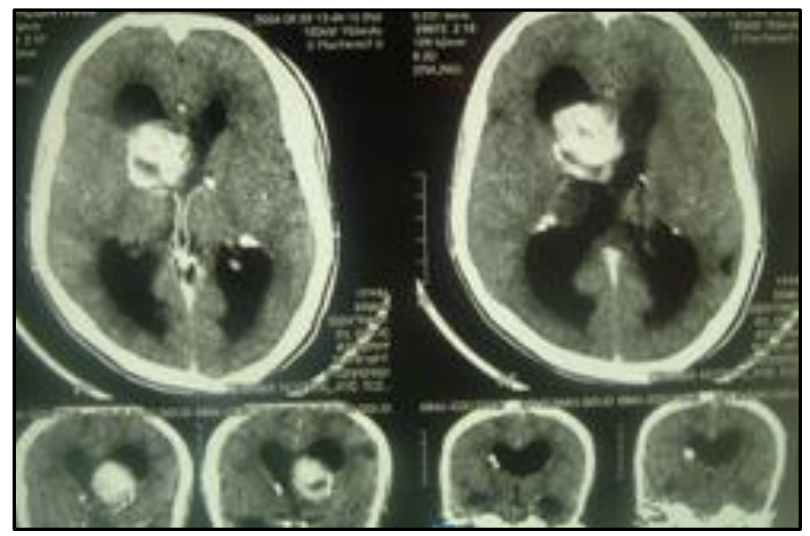

Fig. 1: VEP showing Poor Wave Form

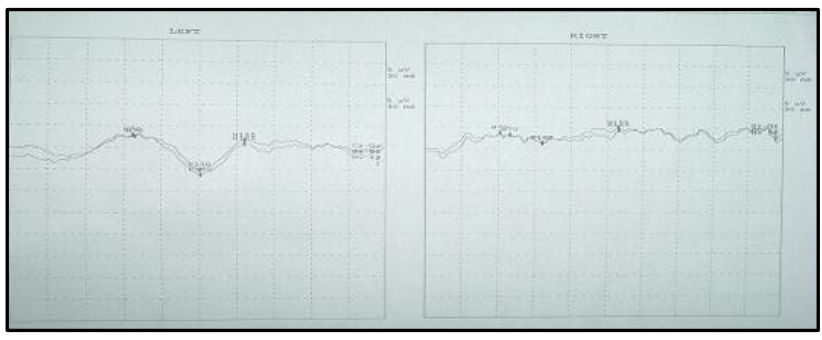

Fig. 2: VEP Prolonged P100 Latency

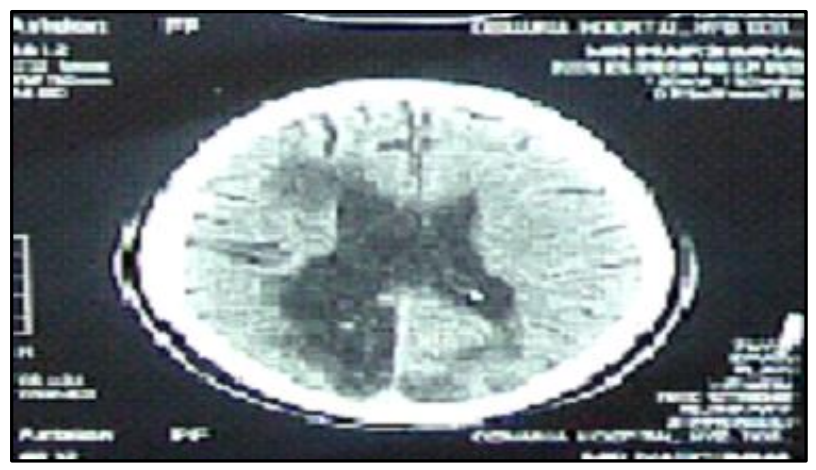

Fig. 3: CT Scan Brain showing Right PCA Infarct
Fig. 5: CT Scan Brain showing Subependymal Mass

\section{DISCUSSION}

Visual loss is a symptom, which generally requires immediate attention of the patient as well as the treating doctor. If there is no trauma, no associated neurological signs or symptoms, the subject will approach an ophthalmologist initially. Subtle visual field defects and slowly progressive visual loss may remain undetected for a long time. The diagnosis of acute optic neuritis is to be made as early as possible and treatment must be instituted on an emergency basis. Different types of steroid protocols were followed by ophthalmologists and neurologists. Beck and Cleary et al had concluded that intravenous high dose methylprednisolone for 3 days followed by 11 days of oral prednisolone offers the best outcome at 6 months after the initial insult, 2,3,4,5,6,7,8,9,10 and also 2 years after the treatment. ${ }^{11}$ In our series, the patients treated with intravenous methylprednisolone made a good recovery compared to dexamethasone or oral steroid therapy Magnetic Resonance Imaging (MRI), serologic studies (Such as the antinuclear antibody test and the fluorescent treponemal antibody absorption test), chest roentgenography and lumbar puncture were of limited utility in defining the cause for visual loss other than Optic Neuritis (ON) associated with demyelinating disease. ${ }^{3}$ CSF analysis may not be necessary in the routine evaluation of patients presenting with a typical clinical profile of acute $\mathrm{ON}$, and that most CSF tests add little additional information to MRI results for predicting the 2-year development of Clinically Definite Multiple Sclerosis (CDMS). ${ }^{12}$ 
Hence, the diagnosis of optic neuritis remains mainly clinical. According to one study, at baseline, contrast sensitivity had the highest prevalence of abnormality in a case of $\mathrm{ON}$, but all vision tests were so often abnormal that differences were not clinically relevant. At six months when visual recovery had occurred, contrast sensitivity was the most abnormal deficit. 13 The recovery of visual function was assessed again 5 years after the initial insult and the risk factors for development of Multiple Sclerosis (MS) were assessed. ${ }^{14,15}$ Those patients with MRI abnormalities in the brain at the time of initial episode of optic neuritis had more chances of developing MS. Our followup was not long and there were no cases of MS in our series. Though we had not encountered any corticosteroid resistant or corticosteroid dependant cases of optic neuritis in the present series, there are studies in which this subject was addressed. Systemic immunosuppression with methotrexate and azathioprine may be a safer and more effective treatment alternative to chronic oral corticosteroid use in cases of corticosteroid dependent optic neuritis not associated with demyelinating disease. ${ }^{16}$ Chronic Relapsing Inflammatory Optic Neuropathy (CRION) was described by Kidd et al with a median followup of 8 (2-26) years and in no case there was evidence for systemic sarcoidosis, tuberculosis or any other granulomatous inflammation. ${ }^{17}$

Most patients retained good-to-excellent vision more than 10 years after an attack of optic neuritis in many studies. Recurrences were more frequent in patients with MS and the recovery from an episode was also less optimal in patients with MS when compared to patients who did not progress to MS. ${ }^{15,18}$ Arnold had opined that in those patients where risk factors for development of MS can be identified, corticosteroids along with immunomodulating drugs may be given to prevent the progression to CDMS. 19

The affected optic nerve swelling persists for a long-time following an attack of optic neuritis and leads to axonal disruption, which results in permanent visual damage. The extent of such residual visual failure depends on the extent of initial damage. In the past, these changes were monitored by imaging techniques. ${ }^{20,21,22}$

Optic neuritis is a disease of younger population, and majority of our patients too were in the age group of 10-19 years. In a study by Morales et al, children had bilateral disease more often than in adults, prognosis for visual function recovery was poorer, and nearly $26 \%$ went on to develop MS. ${ }^{23}$ Most of the ophthalmologists and neurologists would treat patients with ON with intravenous methylprednisolone. Any atypical presentations should prompt one to investigate further to exclude secondary causes of visual failure.24,25,26 Indian's experience with optic neuritis is not very wide, but is consistent with the studies elsewhere. 27

In a long-term followup of recurrent ON, Pirko et al observed that $12.5 \%$ patients developed Neuromyelitis Optica (NMO) and another $14.4 \%$ developed MS. They had also found that the final visual impairment was greatest in NMO group. They concluded that rapid succession of severe ON events are more likely to develop into a generalised demyelinating disease.28 Our followup had not been that long and in the limited followup of our patients of less than 1 year duration there was residual visual impairment, which was not very severe.
A variety of tumours and pseudo-tumours can involve the orbit and can affect the visual function. In a large study involving 1264 cases, the incidence of tumours was as follows: $17 \%$ lymphomas, leukaemias $10 \%$, metastatic lesions $11 \%$ and inflammatory masses in $11 \%$ cases. ${ }^{29}$ There were no orbital masses identified in the present study.

Stroke was the commonest cause of sudden visual impairment seen in nearly $75 \%$ of our cases. Many such patients had binocular visual impairment; one had homonymous hemianopia contralateral to the side of the lesion. Some had altitudinal defects. Brain tumours produced chronic and progressive visual impairment. Some patients with demyelinating disease also had chronic visual involvement. Traumatic visual loss, though not included in our cases, can also form a significant proportion of the patient volume. Many types of visual impairment are described secondary to trauma, ranging from complete loss of vision due to anterior visual pathway injury to isolated colour blindness (Acquired cerebral achromatopsia) due to visual cortex trauma. ${ }^{30}$

The most common aetiology of acquired cerebral achromatopsia is embolic stroke in Posterior Cerebral Artery (PCA) territory. ${ }^{31}$ We did not come across of this kind of isolated defect in our stroke patients. Prosopagnosia is another residual defect, which carries a poor prognosis. The anatomical substrate for prosopagnosia is variably described as left occipitotemporal area, right fusiform gyrus or a combination of these. Stroke patients are known to be left with prosopagnosia as residual deficit and specific localised lesions of these areas had been identified with this problem. ${ }^{32,33}$ Balint's syndrome, a triad of simultagnosia, ocular apraxia and optic ataxia is described as a result of trauma, embolic stroke or even sub-acute HIV encephalitis. ${ }^{34}$ Palinopsia (Perseveration of visual image even after the causative object is removed from the visual field otherwise described as 'persisting after-images') can result from right occipital damage due to stroke, trauma or tuberculoma. ${ }^{35}$

The mechanism of palinopsia is still unclear whether it is an epileptic phenomenon or a release phenomenon. Micropsia, perceiving objects as smaller than their original size, can occur due to cerebrovascular disease, 36 psychogenic conditions or in foveal diseases wherein the symptom is unilateral. Hallucinations in the form of Charles Bonnet syndrome (Formal, complex, persistent, stereotypical visual hallucinations) were described in a 56-year-old woman with multiple sclerosis, but without any cognitive impairment or any comorbid psychiatric symptoms. ${ }^{37}$

Visual field defects particularly homonymous hemianopia, are common following stroke including our own data. Vision recovers in the hemianopic field in stages starting with perception of light and followed by motion, form, colour and stereognosis in that order. ${ }^{38}$ Less than $10 \%$ of the patients recover their full visual field. A variety of different approaches including optical aids and visual training techniques have been examined for the rehabilitation of these field defects. Compensatory visual field training with use of exploratory eye movements, rather than with eyes fixed, had shown considerable improvement in object detection and reaction time. ${ }^{39,40}$ Pambakian et al had shown that hemianopic patients can improve their visual search with continued practice. They had 29 patients practice visual search paradigms for one month and had statistically significant improvement in their 
visual search and reaction time.41 Wang described a 65-year lady with right hemianopia who used a trick to rotate the article by 90 degrees and read it up to down instead of the usual left to right manner. ${ }^{42}$ The sophisticated training techniques are still in the experimental stage. We had not used any of these techniques in our patients.

\section{CONCLUSIONS}

1. The commonest cause of acute unilateral and bilateral visual loss was demyelinating optic neuropathy.

2. Chronic visual loss can also be due to demyelinating optic neuropathy as seen in two patients.

3. The patients with acute and sub-acute visual loss without any identifiable cause can be empirically treated with steroids if not contraindicated.

4. Intravenous methylprednisolone for 3 days followed by 11 days of oral prednisolone is the best mode of treatment for demyelinating optic neuropathy.

5. Recurrence of optic neuropathy was not seen during the follow-up period of the study.

6. Stroke is the commonest cause of sudden visual impairment.

7. The visual loss due to stroke has poor prognosis and the treatment of hemianopia due to stroke is still in the experimental stage only.

8. Stroke can also result in only colour blindness, prosopagnosia or micropsia without there being a total blindness.

9. Slowly progressive visual loss needs investigatory workup to exclude intracranial tumour.

\section{LIMITATIONS OF THE STUDY}

1. Contrast MRI and fat-suppressed imaging are better options to evaluate optic neuritis cases, especially the atypical ones. Due to financial constraints, MRI could be done only in 12 patients in whom one patient showed demyelinating features in the cerebral white matter. Cranial CT scan is used to exclude space occupying lesions and is insensitive to white matter changes or optic nerve enlargement and/or oedema.

2. An attempt was made to identify mitochondrial disorders by way of cerebrospinal fluid, lactate levels estimation and muscle biopsy, but could be done in 7 patients only. It was difficult to convince the patients for invasive procedures on the skeletal muscle when the symptom was affecting some other organ (Eye) in the body. The tests done in a few cases, however, were negative for mitochondrial disease.

3. Recurrence was not seen in any patient, but it would be premature to conclude this way as the follow-up period was less than a year in many patients in the present series. The literature shows that recurrence of optic neuritis increases with the duration of follow-up, particularly in patients with white matter abnormalities in the brain.

\section{REFERENCES}

1. World Health Organization report. Magnitude and causes of visual impairment 2004.

2. Beck RW. The optic neuritis treatment trial. Arch Ophthalmol 1988;106(8):1051-3.
3. The clinical profile of acute optic neuritis: experience of the optic neuritis treatment trial. Optic neuritis study group. Arch Ophthalmol 1991;109(12):1673-8.

4. Beck RW, Cleary PA, Anderson MM, et al. A randomized, controlled trial of corticosteroids in the treatment of acute optic neuritis. New Eng J Med 1992;326(9):581-8.

5. Beck RW, Kupersmith MJ, Cleary PA, et al. Fellow eye abnormalities in acute unilateral optic neuritis: experience of the optic neuritis treatment trial. Ophthalmology 1993;100(5):691-8.

6. Keltner JL, Johnson CA, Spurr JO, et al. Baseline visual field profile of optic neuritis: the experience of the optic neuritis treatment trial. Optic neuritis study group. Arch Ophthalmol 1993;111(2):231-4.

7. Beck RW, Arrington J, Murtagh FR, et al. Optic neuritis study group. Brain MRI in acute optic neuritis: experience of the optic neuritis study group. Arch Neurol 1993;50(8):841-6.

8. Cleary PA, Beck RW, Anderson MM, et al. Design, methods, and conduct of the optic neuritis treatment trial. Controlled Clinical Trials 1993;14(2):123-42.

9. Beck RW, Cleary PA. Optic neuritis study group. Recovery from severe visual loss in optic neuritis. Arch Ophthalmol 1993;111(3):300.

10. Beck RW, Cleary PA, Trobe JD, et al. The effect of corticosteroids for acute optic neuritis on the subsequent development of multiple sclerosis. Optic neuritis study group. New Eng J Med 1993;329(24):1764-9.

11. Beck RW. Optic neuritis study group. The optic neuritis treatment trial: three-year follow-up results. Arch Ophthalmol 1995;113(2):136-7.

12. Rolak LA, Beck RW, Paty DW, et al. Cerebrospinal fluid in acute optic neuritis: experience of the optic neuritis treatment trial. Neurology 1996;46(2):368-72.

13. Trobe JD, Beck RW, Moke PS, et al. Contrast sensitivity and other vision tests in the optic neuritis treatment trial. Am J Ophthalmol 1996;121(5):547-53.

14. Optic Neuritis Study Group. The five year risk of MS after optic neuritis. Experience of the optic neuritis treatment trial. Neurology 1997;49(5):1404-13.

15. Optic Neuritis Study Group. Visual function five years after optic neuritis. Experience of the optic neuritis treatment trial. Arch Ophthalmol 1997;115(12):1545-52.

16. Myers TD, Smith JR, Wertheim MS, et al. Use of corticosteroid sparing systemic immune-suppression for treatment of corticosteroid dependent optic neuritis not associated with demyelinating disease. $\mathrm{Br} \mathrm{J}$ Ophthalmol 2004;88(5):673-80.

17. Kidd D, Burton B, Plant GT, et al. Chronic relapsing inflammatory optic neuropathy. Brain 2003;126(Pt2):276-84.

18. Beck RW, Gal RL, Bhatti MT, et al. Visual function more than 10 years after optic neuritis: experience of the optic neuritis treatment trial. Am J Ophthalmol 2004;137(1):77-83.

19. Arnold AC. Evolving management of optic neuritis and multiple sclerosis. Am J Ophthalmol 2005;139(6):1101-8.

20. Chabert S, Molko N, Cointepas Y, et al. Diffusion tensor imaging of the human optic nerve using a non-CPMG fast spin echo sequence. J Magn Reson Imaging 2005;22(2):307-10. 
21. Hickman SJ, Wheeler-Kingshott CA, Jones SJ, et al. Optic nerve diffusion measurement from diffusion-weighted imaging in optic neuritis. AJNR Am J Neuroradiol 2005;26(4):951-6.

22. Killer HE, Mironov A, Flammer J. Optic neuritis with marked distension of the optic nerve sheath due to local fluid congestion. Br J Ophthalmol 2003;87(2):249.

23. Morales DS, Siatkowski RM, Howard CW, et al. Optic neuritis in children. J Pediatr Ophthalmol Strabismus 2000;37(5):254-9.

24. Lee AG, Lin DJ, Kaufman M, et al. A typical features prompting neuroimaging in acute optic neuropathy in adults. Can J Ophthalmol 2000;35(6):325-30.

25. Ghosh A, Kelly SP, Mathews J, et al. Evaluation of the management of optic neuritis: audit on the neurological and ophthalmological practice in the north west of England. J Neurol Neurosurg Psychiatry 2002;72(1): 119-21.

26. Bradley WG, Daroff RB, Fenichel GM, et al. Neurology in clinical practice. $4^{\text {th }}$ edn. London: Elsevier Health Sciences 2004.

27. Wadia NH. Neurological practice- an Indian perspective. Published by Elsevier, New Delhi 2005:458.

28. Pirko L, Blauwet LA, Lesnick TG, et al. The natural history of recurrent optic neuritis. Arch Neurol 2004;61(9):1 401-5.

29. Shields AJ, Shields CL, Scartozzi R. Survey of 1,264 patients with orbital tumors and simulating lesions: the 2002 Montgomery Lecture, part 1. Ophthalmology 2004;111(5):997-1008.

30. Sacks O, Wasserma R. The case of the color blind painter. New York: Alfred A. Knopf 1995.

31. Spillman L, Laskowski W, Lange KW, et al. Stroke-blind for colours, faces and locations: partial recovery after three years. Restor Neurol Neurosci 2000;17(2-3):89-103.
32. Barton JJ, Cherkasova M. Face imagery and its relation to perception and covert recognition in prosopagnosia. Neurology 2003;61(2):220-5.

33. Wada Y, Yamamoto T. Selective impairment of facial recognition due to a haematoma restricted to the right fusiform and lateral occipital region. J Neurol Neurosurg Psychiatry 2001;71(2):254-7.

34. Schneider A, Landis T, Regard M. Balint's syndrome in sub-acute HIV encephalitis. J Neurol Neurosurg Psychiatry 1991;54(9):822-5.

35. Werring DJ, Marsden C. Visual hallucinations and palinopsia due to an occipital lobe tuberculoma. J Neurol Neurosurg Psychiatry 1999;66(5):684.

36. Ceriani F, Gentileschi V, Muggia S, et al. Seeing objects smaller than they are: micropsia following right temporoparietal infarction. Cortex 1998;34(1):131-8.

37. Chen CS, Lin SF, Chong MY. Charles bonnet syndrome and multiple sclerosis. Am J Psychiatry 2001;158(7):1158-9.

38. Rossell RR. The posterior cerebral circulation. J Royal Coll Phys 1973;7:331-46.

39. Pambakian A, Currie J, Kennard C. Rehabilitation strategies for patients with homonymous visual field defects. J Neuroophthalmol 2005;25(2):136-42.

40. Nelles G, Esser J, Eckstein A, et al. Compensatory visual field training for patients with hemianopia after stroke. Neurosci Lett 2001;306(3):189-92.

41. Pambakian AL, Mannan SK, Hodgson TL, et al. Saccadic visual search training: a treatment for patients with homonymous hemianopia. J Neurol Neurosurg Psychiatry 2004;75(10):1443-8.

42. Wang MK. Reading with a right homonymous hemianopia. Lancet 2003;361(9363):1138. 